\title{
Original
}

\section{Hábitos sexuales en los adolescentes de 13 a 18 años}

\author{
M. Alfaro González ${ }^{a}$, M. E. Vázquez Fernández ${ }^{b}$, A. Fierro Urturic, M. F. Muñoz Morenod, \\ L. Rodríguez Molinero ${ }^{\mathrm{e}}$, C. González Hernando ${ }^{\mathrm{f}}$, Grupo de Educación para la Salud de la AEPap
}

Publicado en Internet:

9-septiembre-2015

María Alfaro González: mariaalfaro28@hotmail.com

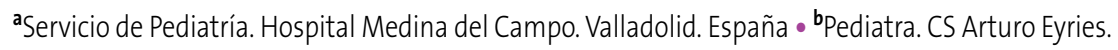
Valladolid. España • Pediatra. CS Pisuerga. Valladolid. España • dUnidad de Investigación Biomédica. Hospital Clínico Universitario de Valladolid. España • ${ }^{\mathrm{e} P e d i a t r a . ~ C S ~ C a s a ~ d e l ~ B a r c o . ~ V a l l a d o l i d . ~ E s p a n ̃ a ~ • ~}{ }^{{ }^{E} \text { Enfermera }}$ especialista/matrona. Profesora. Facultad de Enfermería de la Universidad de Valladolid. España.

Introducción: la población adolescente es especialmente vulnerable a los riesgos relacionados con su conducta sexual, dado que se trata de un periodo de maduración en el que experimentar forma parte de esta etapa evolutiva. El embarazo en adolescentes y la maternidad temprana están asociados con el fracaso escolar, el deterioro de la salud física y mental, aislamiento social, pobreza y otros factores relacionados. Por otra parte, no usar métodos anticonceptivos de barrera aumenta el riesgo de infecciones de transmisión sexual (ITS), con serias consecuencias a corto y largo plazo en la salud biopsicosocial de los adolescentes.

Objetivos: conocer las características sobre sexualidad de los adolescentes de la provincia de Valladolid. Métodos: estudio transversal descriptivo mediante encuesta anónima a una muestra de 2412 escolares de 13 a 18 años, en el curso académico 2011-2012.

Resultados: el $81 \%$ de los alumnos consideran que tienen una información muy buena o buena sobre sexualidad. El $75 \%$ de los alumnos consideran que la información que tienen sobre infecciones de transmisión sexual es muy buena o buena. Han tenido relaciones con penetración el 30,4\% de los alumnos. La edad de inicio de las relaciones sexuales completas se sitúa entre los 15 y 16 años (50,9\%). La mayoría declaran que utilizan algún método anticonceptivo en sus relaciones (91,3\%), pero todavía hay un $8,7 \%$ que refiere no utilizar ninguno. La mayoría utilizan como método anticonceptivo el preservativo (89,6\%). Un 20,9\% de los adolescentes que mantienen relaciones sexuales con penetración han utilizado en alguna ocasión la píldora del día después o anticoncepción de urgencia. El 3,6\% declara haberse quedado embarazada o haber dejado embarazada a su pareja.

Conclusiones: a pesar de considerarse suficientemente informados sobre sexualidad e ITS, los adolescentes presentan comportamientos de riesgo en sus actividades sexuales.

\section{Sexual behavior in adolescents 13 to 18 years old}

Introduction: the adolescent population is particularly vulnerable to the risks related with their sexual behavior, because it is a period of maturation and experience which is part of this evolutionary stage. Teenage pregnancy and early motherhood are associated with school failure, impaired physical and mental health, social isolation, poverty and other related factors. Moreover the non-use of appropriate protective barriers increases the risk of sexually transmitted infections (STI), with serious consequences in the short and long term biopsychosocial adolescent health.

Objectives: to determine the characteristics of adolescent sexuality in the province of Valladolid.

Methods: cross-sectional study, using an anonymous survey to 2412 schoolchildren 13-18 years old in the academic year 2011-2012.

Results: $81 \%$ of students feel they have very good or good information on sexuality. $75 \%$ of students believe that the information they have about STI is very good or good. $30.4 \%$ have had sexual intercourse. The age of first complete sexual intercourse is between 15 and 16 years (50.9\%). Most of the adolescents use a contraceptive method in their relationships (91.3\%), but there are still $8.7 \%$ referring not using any. Most of the students use the condom for contraceptive method (89.6\%). $20.9 \%$ of teens who have had sex with penetration have used "the morning-after pill" or emergency contraception at some time. $3.6 \%$ reported having being pregnant or having left his partner pregnant.

Key words:

- Adolescent

- Sexuality

- Pregnancy

- Contraceptive methods

Conclusions: although they consider themselves sufficiently informed about sexuality and STI, adolescents present risk behaviors in their sexual activities. 


\section{INTRODUCCIÓN}

Alcanzar conductas responsables relacionadas con la sexualidad, con todas sus implicaciones físicas y psicológicas, es una de las tareas a las que debe hacer frente el adolescente. Se trata de un periodo de maduración en el que experimentar es algo normal. En el informe final donde se explicitan las principales directrices de la política sanitaria europea para las primeras dos décadas del siglo XXI, consensuadas en la 51. a Asamblea Mundial de la Organización Mundial de la Salud (OMS), celebrada en mayo de $1998^{1}$, la comunidad sanitaria consideró a la población adolescente especialmente vulnerable a los riesgos relacionados con su conducta sexual y se hicieron las siguientes consideraciones, aún plenamente vigentes:

- La actividad sexual sin protección genera un gran número de embarazos no deseados, de abortos y de infecciones de transmisión sexual (ITS), incluida la infección por el virus de la inmunodeficiencia humana (VIH).

- En muchas partes no se está suministrando una educación sexual objetiva y sin prejuicios, ni en los centros educativos ni en otros lugares.

- La falta de información y conocimientos sobre cuestiones relativas a la sexualidad, los cambios y funciones corporales y los sentimientos $y$ emociones deriva en una tensión emocional innecesaria.

- Hay una insuficiencia de servicios sanitarios de carácter confidencial pensados para jóvenes. Esta carencia puede limitar su acceso a una atención y un asesoramiento adecuados.

En los países desarrollados, una alta proporción de jóvenes que finalizan la escuela obligatoria informan de haber participado en comportamientos sexuales de riesgo ${ }^{2,3}$. Las relaciones sexuales desprotegidas o protegidas de manera incorrecta conllevan riesgo de embarazos no deseados, con las consiguientes consecuencias negativas para este grupo de edad, como el aborto, la maternidad precoz o la adopción ${ }^{4}$. El embarazo en adolescentes y la maternidad temprana están asociados con el fracaso escolar, el deterioro de la salud física y mental, el aislamiento social, la pobreza y otros factores relacionados. Además el no uso de adecuadas barreras de protección aumenta el riesgo de infecciones de transmisión sexual, con consecuencias a corto y largo plazo en la salud biopsicosocial de los adolescentes ${ }^{5}$.

Por todo ello, abordar la salud sexual de los adolescentes mediante el aumento de su compromiso con el sexo seguro se ha convertido en un problema importante en los países desarrollados ${ }^{5,6}$.

\section{MATERIAL Y MÉTODOS}

\section{Población de referencia y marco muestral}

La población de referencia han sido estudiantes que cursan Enseñanzas Secundarias de 13 a 18 años. La base utilizada para seleccionar la muestra fue la población matriculada en colegios o centros educativos con 2.․ 3.․ y 4.을 de Enseñanza Secundaria Obligatoria (ESO), 1.ำ y $2 . \circ$ de Bachillerato LOGSE. Este marco condiciona la distribución de la muestra por edad.

En este trabajo no se incluyeron los estudiantes de 13-18 años que estaban cursando Educación Primaria o Educación Universitaria o Formación Profesional, los que no asistieron a clase el día y hora en que se aplicó el cuestionario (ausentes), los de Enseñanzas de Régimen General incluidos en Programas de Garantía Social y a distancia, de Enseñanzas de Régimen Nocturno y de Enseñanzas de Régimen Especial.

\section{Muestra}

La selección de alumnos se realizó mediante muestreo bietápico por conglomerados, seleccionando aleatoriamente colegios ( $n=14)$ en una primera etapa y aulas en la segunda. Posteriormente se incluyó a todos los estudiantes de las aulas seleccionadas.

El tamaño muestral se calculó para una proporción estimada de un $50 \%$ y una precisión del 2,5\% en un contraste bilateral, asumiendo un 10\% de falta de 
respuestas, resultando 1566 alumnos. El número final de alumnos encuestados superó claramente lo esperado, después de depurar y eliminar encuestas que no estaban cumplimentadas en su totalidad; la muestra final fue de 2412 adolescentes escolarizados de 13 a 18 años.

\section{Cuestionario y trabajo de campo}

El instrumento de recogida de datos utilizado fue un cuestionario con 101 preguntas adaptado y similar al de otros estudios internacionales ${ }^{7,8}$, nacionales ${ }^{9,10}$ y de distintas comunidades autónomas y provincias $^{11-15}$, ya validados.

Se realizaron preguntas relacionadas con variables sociodemográficas, rendimiento escolar, ocio y tiempo libre, accidentes, tabaco, alcohol, drogas, conductas, experiencia sobre el maltrato y relaciones con los demás, alimentación y sexualidad.

Se cumplimentó de manera anónima, individual y voluntaria, durante el horario de clase: asistido por ordenador en un 69\% de los casos y por escrito (papel y lápiz) en el 31\% restante. El tiempo empleado para responder al cuestionario fue de 35-40 minutos.

El equipo investigador se encargó de administrar la encuesta, ayudado en ocasiones por el profesorado. Todas las encuestas se realizaron entre marzo y mayo de 2012.

El diseño del proyecto fue aprobado por la Comisión de Investigación de la Gerencia de Atención Primaria del Área Oeste de Valladolid.

\section{Análisis estadístico}

Las variables cuantitativas se presentan con los valores medios y el intervalo de confianza del $95 \%$ y las cualitativas según su distribución de frecuencias.

Los datos han sido analizados con el programa estadístico SPSS ${ }^{\circledR}$ versión 19.0 para Windows ${ }^{\circledR}$. Los valores de $p<0,05$ han sido considerados estadísticamente significativos.

\section{RESULTADOS}

De los alumnos encuestados, fueron validadas un total de 2412 encuestas; el 47,3\% corresponden a mujeres y el $52,7 \%$ a varones. En relación al curso, un $23,5 \%$ pertenecen a $2 .{ }^{\circ}$ de la ESO; un $25,8 \%$ a 3. de la ESO; un $20,2 \%$ a 4. . de la ESO; un $18,9 \%$ a 1.ㅇ de Bachillerato y un $11,6 \%$ a $2 . . \circ$ de Bachillerato.

\section{Grado de información sobre sexualidad}

El $81 \%$ de los alumnos consideran que tienen una información muy buena o buena sobre sexualidad. Los chicos se consideran mejor informados que las chicas (el 84,1\% frente al 77,5\%) ( $p<0,001$ ). Solo un $3,9 \%$ considera que está poco o nada informado sobre sexualidad, siendo los menores de 2.ํ de la ESO los que en mayor frecuencia refieren estar poco o nada informados (el 7,7\% frente al 2,2\% de 1.ㅇ de Bachillerato) $(p<0,001)$.

\section{Grado de información sobre enfermedades de transmisión sexual}

El 75\% de los alumnos consideran que la información que tienen sobre enfermedades de transmisión sexual es muy buena o buena, siendo este porcentaje mayor en chicos $(78,2 \%)$ que en chicas $(71,7 \%)(p<0,001)$. Sin embargo, un $6,1 \%$ de los alumnos consideran que la información que tienen sobre enfermedades de transmisión sexual (ETS) es poca o nula. Son los alumnos de cursos inferiores los que en mayor medida se consideran que tienen insuficiente información (el 12,8\% en 2. de la ESO frente al 3,2\% en 2. de Bachillerato) ( $p<0,001)$.

\section{Orientación sobre sexualidad}

A la pregunta de si necesitasen orientación sobre sexualidad, con quién hablarían, un 59,3\% hablaría con sus amigos y el $51,7 \%$ con sus padres. En tercer lugar buscarían información en Internet, el 27,8\%. En cuarto lugar, recurrirían a profesionales sanitarios, el 19,9\%, sin diferencias entre chicos y chicas. En menor medida hablarían con sus profesores (8,8\%), hablarían con otras personas el 5,2\% y un $7,7 \%$ refiere que no hablaría con nadie.

\section{Frecuencia de relaciones sexuales coitales}

Han tenido relaciones con penetración alguna vez en su vida el 30,4\% de los alumnos. El porcentaje 
de chicos que dice haber mantenido relaciones sexuales coitales $(32,4 \%)$ es superior que el de las chicas $(28,2 \%)(p=0,028)$. A medida que avanza el curso, aumenta la frecuencia de los que han tenido relaciones, así el 13,1 \% de los alumnos de 2. de la ESO han tenido relaciones con penetración frente al $63,1 \%$ de 2.․ de Bachillerato ( $p<0,001$ ) (Tabla 1).

Declaran haber tenido solo una vez relaciones completas el 5,2\%, algunas veces al año (menos de una vez al mes) el 8,25\%, varias veces al mes el $11,13 \%$ y varias veces por semana el $5,82 \%$ de los alumnos.

\section{Edad de inicio de relaciones sexuales coitales}

La edad de inicio de las relaciones sexuales completas se sitúa entre los 15 y 16 años (50,9\%). Es significativa la mayor frecuencia de inicio de las relaciones en este intervalo de edad de las chicas $(58 \%)$ frente a los chicos $(45,4 \%)(p<0,001)$. Un $18,8 \%$ inician las relaciones entre los 13 y 14 años, por encima de los 17 años el 13,9\% y con menos de 12 años el 8,4\%. La distribución por edad y sexo se muestra en la Fig. 1.

Según curso, es significativo $(p<0,001)$ que los alumnos de cursos inferiores refieran que inician las relaciones completas más precozmente que los alumnos de cursos superiores. Así, el 35,7\% de los alumnos de 2. de la ESO declara que ha tenido su primera relación coital con 12 años o menos frente

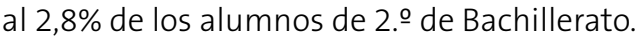

\section{Actividad sexual}

De los que han tenido relaciones sexuales con penetración, las han tenido en el último año el 91,3\%. De ellos han tenido relaciones solo con su pareja el $57 \%$, con dos personas el $14,2 \%$ y con más de dos personas el $20,1 \%$. Es significativo $(p<0,001)$ que son las chicas las que refieren haber tenido en mayor frecuencia relaciones solo con su pareja que los chicos (el 69\% frente al 47,5\%), y son los chicos los que en mayor frecuencia declaran haber tenido más de dos parejas sexuales (el 27,5\% frente al 10,7\%). Según el curso, son los mayores de $2 .^{\circ}$ de Bachillerato los que tienen relaciones más estables, un $63,4 \%$ refieren tener solamente relaciones con su pareja frente al 49,3\% de 2.. de la ESO. Sin embargo, los menores de 2. de la ESO son los que en mayor frecuencia declaran haber tenido relaciones sexuales con dos o más personas (28,8\%), disminuyendo a medida que avanza el curso (el 13,7\% en 2.ํ de Bachillerato).

\section{Métodos anticonceptivos}

La mayoría declara que utilizan algún método anticonceptivo en sus relaciones (91,3\%), pero hay un $8,7 \%$ que refiere no utilizar ninguno. No hay diferencias según el sexo. Los alumnos que en mayor frecuencia no han utilizado ningún método anticonceptivo son los de 3. o de la ESO (15,8\%), seguidos de los de 2. de la ESO (12,3\%), 2. de Bachillerato $(7,4 \%), 4 .$. de la ESO (6\%) y 1. de Bachillerato $(5,7 \%)(p=0,009)$.

La mayoría utiliza como método anticonceptivo el preservativo $(89,6 \%)$. No hay diferencias significativas según el sexo. Si hay diferencias $(p=0,002)$ en relación al curso, aumentando su uso según avanza la edad.

Han utilizado la píldora anticonceptiva el 8,6\% de los alumnos. No hay diferencias estadísticamente significativas según el sexo y curso.

Utilizan el cálculo de la fecha de ovulación el 1,5\% y la marcha atrás el 9,5\%. En menor medida utilizan espermicidas (1\%), el diafragma $(0,8 \%$ ) y el dispositivo intrauterino $(0,6 \%)$. Refieren que utilizan otros métodos anticonceptivos el 3\%.

Tabla 1. Adolescentes que han mantenido relaciones sexuales coitales según sexo y curso

\begin{tabular}{|l|c|c|c|c|c|c|c|c|c|}
\hline \multirow{2}{*}{} & \multicolumn{2}{|c|}{ Total } & \multicolumn{2}{c|}{ Sexo (\%) } & \multicolumn{5}{c|}{ Edad (\%) } \\
\cline { 2 - 11 } & Número & $\%$ & Chico & Chica & 2. . ESO & $3 .$. ESO & 4. . ESO & 1. o Bachillerato & 2. o Bachillerato \\
\hline Sí & 726 & $30,4 \%$ & 32,4 & 28,2 & 13,1 & 21,8 & 34,5 & 38,9 & 63,1 \\
\hline No & 1663 & $69,6 \%$ & 67,6 & 71,8 & 86,9 & 78,2 & 65,5 & 61,1 & 36,9 \\
\hline
\end{tabular}




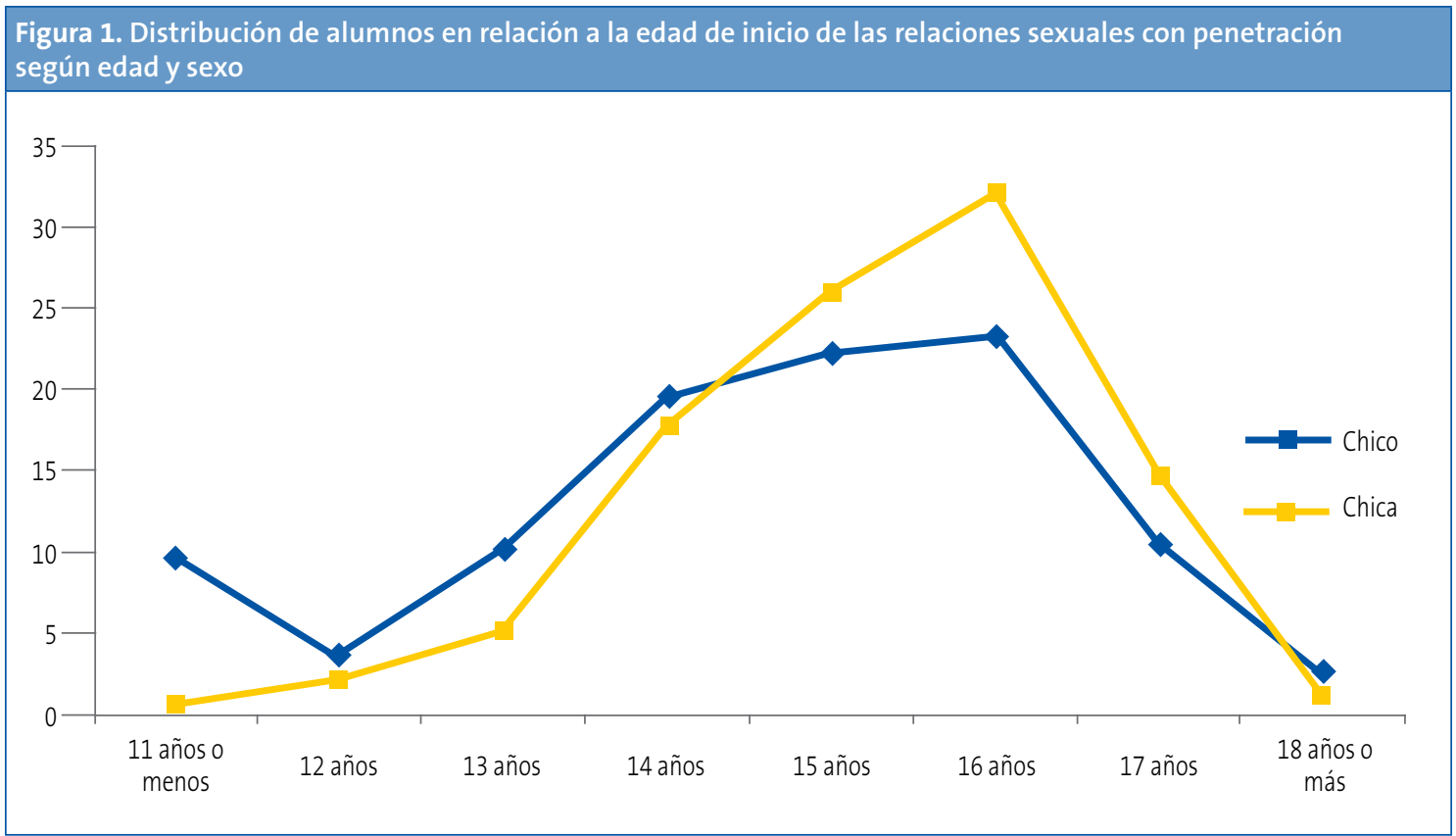

A la pregunta sobre si han rechazado una relación sexual si no disponían de un preservativo, un 58,8\% contesta afirmativamente, siendo significativo $(p<0,001)$ la mayor proporción de chicas que lo hacen $(71,7 \%)$ frente a los chicos (48,5\%). Esta proporción es mayor en alumnos de 1.ำ de Bachillerato $(65,3 \%)$ y 2. o de Bachillerato $(61,5 \%)$ que en los de

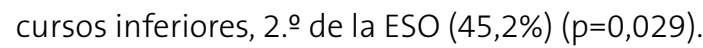

Un 20,9\% de los adolescentes que mantienen relaciones sexuales con penetración han utilizado en alguna ocasión la píldora del día después; un $16,1 \%$ lo han hecho en los últimos seis meses y un $3 \%$ ha recurrido a la anticoncepción de emergencia en más de una ocasión en los últimos seis meses.
En cuanto a la distribución por cursos, son los alumnos de 2. de la ESO los que declaran haber recurrido en mayor porcentaje a este método (31,5\%), seguidos de los de 2.ํ de Bachillerato (24\%), 1. de Bachillerato (20,7\%), 3. de la ESO (18\%) y 4. . - de la $\operatorname{ESO}(15,6 \%)(p=0,05)$. En cuanto al uso en los últimos seis meses, es significativo el mayor porcentaje en los alumnos de cursos inferiores, tanto una vez como más de una, como se muestra en la Tabla 2.

\section{Embarazo}

El 3,6\% declara haberse quedado embarazada o haber dejado embarazada a su pareja (5,3\% de los

Tabla 2. Uso de la píldora del día después en los últimos seis meses según curso entre los adolescentes que declaran mantener relaciones sexuales con penetración

\begin{tabular}{|c|c|c|c|c|c|c|}
\hline \multirow{2}{*}{\multicolumn{2}{|c|}{$\begin{array}{l}\mathrm{p}<0,001 \\
\text { 2. } \mathrm{ESO}\end{array}$}} & \multicolumn{5}{|c|}{ Curso } \\
\hline & & \multirow{2}{*}{$\begin{array}{c}3.9 \text { ESO } \\
1\end{array}$} & \multirow{2}{*}{ 4. ESO } & \multirow{2}{*}{ 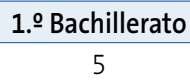 } & \multirow{2}{*}{$\begin{array}{c}\text { 2.9 Bachillerato } \\
10\end{array}$} & \multirow[b]{2}{*}{17} \\
\hline Ninguna & Recuento & & & & & \\
\hline & $\%$ & $4,3 \%$ & $4,3 \%$ & $19,2 \%$ & $27,0 \%$ & $40,5 \%$ \\
\hline \multirow[t]{2}{*}{ Una } & Recuento & 14 & 17 & 17 & 24 & 23 \\
\hline & $\%$ & $60,9 \%$ & $73,9 \%$ & $65,4 \%$ & $64,9 \%$ & $54,8 \%$ \\
\hline \multirow[t]{2}{*}{ Más de una } & Recuento & 8 & 5 & 4 & 3 & 2 \\
\hline & $\%$ & $34,8 \%$ & $21,7 \%$ & $15,4 \%$ & $8,1 \%$ & $4,8 \%$ \\
\hline
\end{tabular}


chicos y $1,3 \%$ de las chicas). Es significativa $(p<0,001)$ la mayor proporción de alumnos de 2. o de la ESO que lo refieren (9,9\%), disminuyendo en los alumnos de cursos superiores (el 1,8\% en 2. de Bachillerato). El 11,4\% de los chicos desconoce si ha dejado embarazada a su pareja, y el 3,3\% de las chicas refiere desconocer si está embarazada.

\section{Sexo, drogas y alcohol}

Un 23,4\% declara haber tomado alcohol y/o drogas la última vez que mantuvo relaciones sexuales con penetración. Es significativo $(p<0,001)$ el mayor porcentaje de chicos $(29,2 \%)$ que de chicas $(16 \%)$. No es significativo en relación al curso.

\section{DISCUSIÓN}

La adolescencia se caracteriza por tratarse de un periodo de desarrollo y crecimiento, particularmente en el área de la afectividad y la sexualidad. Aunque el desarrollo afectivo-sexual tiene lugar a lo largo de toda la vida, en esta etapa se dan importantes cambios en la persona. En España, muchos jóvenes comienzan a tener sus primeras relaciones sexuales en estas edades. Esta situación entraña numerosos riesgos para la salud y el bienestar general de estos jóvenes, como pueden ser los embarazos precoces, las infecciones de trasmisión sexual (ITS), las relaciones sexuales forzadas, los sentimientos de decepción o arrepentimiento ante las relaciones sexuales. Los costes emocionales, médicos y económicos de estos riesgos subrayan la importancia de estudiar la iniciación sexual juvenil y sus elementos asociados.

El estudio de la conducta sexual realizado en este trabajo muestra de manera global que casi una tercera parte de los adolescentes (30,4\%) afirma haber mantenido al menos una relación coital en su vida. Este porcentaje se va elevando al aumentar la edad de los encuestados, superando el $60 \%$ en 2. de Bachillerato, observando además mayor incidencia en el sexo masculino. Pese a ello, en aproximadamente la mitad de los alumnos/as se tratan de relaciones esporádicas (una vez en la vida o alguna vez en el año). Datos similares aparecen en el estudio HBSC $^{9}$. En el estudio SIVFRENT ${ }^{16}$, realizado con población adolescente de la Comunidad de Madrid, el porcentaje es ligeramente superior, del 34,6\%, sin encontrar diferencias en cuanto al género. El Observatorio de Salud de Terrasa muestra también porcentajes superiores de relaciones sexuales coitales, situándose en un 36\% en 2010, pero con más frecuencia en el sexo femenino ${ }^{12}$.

En nuestro estudio, de los que han mantenido relaciones sexuales coitales, el 91,3\% dice haber utilizado en su última relación sexual algún método anticonceptivo, especialmente el preservativo. El segundo método anticonceptivo más utilizado es la píldora. El resto de métodos anticonceptivos (diafragma, espermicidas, DIU y otros) se usan en muy baja proporción, quizás por desconocimiento, precio o accesibilidad. Y hay más de un $10 \%$ que utiliza como medio de anticoncepción "la marcha atrás"y/o la fecha de ovulación.

Además destaca que una elevada proporción declara haber usado la anticoncepción de emergencia o "píldora del día después" en los últimos meses, en una o varias ocasiones. La legislación actual permite su adquisición sin prescripción médica a cualquier adolescente, y cada vez es más el número de jóvenes que la utilizan tras mantener relaciones sexuales sin preservativo $u$ otro método anticonceptivo. Lo que no está claro es que la dispensación libre del fármaco haya servido para frenar el número de embarazos no deseados entre las adolescentes, a pesar de que el Ministerio de Sanidad haya anunciado que ha bajado el número de interrupciones del embarazo. En nuestro estudio, los dispares resultados entre chicos y chicas y entre los de menor y mayor edad nos impide corroborar esta situación.

En nuestra encuesta, la frecuencia de uso del preservativo es ligeramente superior a otros estudios nacionales. Los efectos que las campañas de salud sexual han tenido en la frecuencia de uso del preservativo entre los jóvenes han sido notables. Según el Informe de la Juventud en España, puede apreciarse una mayor utilización del preservativo entre los de menor edad ${ }^{17}$. En él se destaca que el 
73,8\% de los jóvenes de 15-17 años utilizaron siempre un preservativo en el último año. Este porcentaje desciende al 64,3\% entre los jóvenes de 18-20 años y al 54,1\% entre los de 21-24 años. Sin embargo, esta notable mejoría en el uso del preservativo parece ir acompañada de un incremento de las conductas sexuales de riesgo juveniles y consumo de alcohol. Uno de cada cuatro mantiene relaciones sexuales bajo los efectos del alcohol o drogas.

Diferentes estudios muestran una tendencia al inicio de las relaciones sexuales con penetración a edades cada vez más tempranas ${ }^{18}$. Según la Encuesta de Salud y Hábitos Sexuales (ESHS) ${ }^{19}$ en España, el 16,5\% de las personas de 18-29 años ha tenido su primera relación sexual con menos de 16 años. De ahí que en los últimos años esté emergiendo un colectivo de interrupción voluntaria del embarazo (IVE) en menores de 16 años, inexistente hasta hace pocos años ${ }^{20}$. En nuestro estudio se confirma esta tendencia, ya que se observa cómo los alumnos de cursos inferiores han iniciado las relaciones sexuales antes que los alumnos de cursos superiores.

Al descender la edad de la primera relación sexual y al retrasarse la edad en la que se forma una familia, aumenta el periodo en el que las y los jóvenes están sexualmente activos, con un mayor número de parejas sexuales. El estilo de vida sexual del o la adolescente y el adulto joven se caracteriza por establecer, en el marco de una relación afectiva, muchas relaciones monógamas a corto plazo. Los y las jóvenes consideran como pareja estable aquella que permanece junta durante un periodo relativamente reducido con respecto a épocas anteriores $^{21}$. En nuestro caso, un 34,3\% han mantenido relaciones sexuales con dos o más parejas en el último año, siendo más frecuente entre los alumnos de 2.ㅁo de la ESO frente a los de 2. de Bachillerato, que ya suelen tener pareja estable. Al analizar las campañas de salud sexual dirigidas a los jóvenes llevadas a cabo desde el Ministerio de Sanidad del Gobierno de España en los últimos años, no hemos encontrado ninguna recomendación en relación a la reducción del número de parejas sexuales $^{22}$. Todas ellas promocionan exclusivamente el uso del preservativo a toda la población.

Existe un alto porcentaje de jóvenes (14\%), similar al resto de estudios, que no emplea métodos seguros para prevenir embarazos no deseados y ITS, incluso más del $8 \%$ que no usan ninguno, lo que pone de manifiesto un desconocimiento sobre medidas de prevención, especialmente en los de menor edad. Estos datos chocan con el hecho de que la mayoría de jóvenes se consideran bien o muy bien informados en materia de sexualidad y en ETS.

Con respecto a las fuentes de información, son los amigos, seguido de los padres y en tercer lugar los medios de comunicación e Internet los medios para obtener información, coincidiendo con otros estu$\operatorname{dios}^{23-25}$. Esto resalta la importancia de cuidar los contenidos de internet y de los medios de comunicación, e indica la efectividad de ofrecerles páginas webs y aplicaciones informáticas seguras para consultar de información, tanto dirigidas a los padres como al propio adolescente, que será sin lugar a duda fuente de difusión, principalmente con amigos.

\section{CONCLUSIONES}

Se debe potenciar la educación sexual integral dirigida a adolescentes, permitiendo que los jóvenes tomen decisiones responsables sobre su salud sexual y no se sientan presionados para iniciar su actividad sexual ni se arriesguen a contraer una ITS o a afrontar un embarazo no deseado ${ }^{26}$. La disminución de problemas como las ITS o los embarazos precoces solo se lograrán a través de una mejora de los estilos de vida, pasando obligatoriamente por la educación sexual. Y esto es improbable que suceda a través de acciones educativas aisladas ${ }^{27,28}$. Parece por tanto necesario un mayor esfuerzo por parte de autoridades sanitarias y profesionales del sector en promover campañas de concienciación y educación sexual entre nuestros jóvenes.

\section{FINANCIACIÓN}

Este trabajo ha sido financiado por la Asociación Española de Pediatría de Atención Primaria (AEPap) 


\section{CONFLICTO DE INTERESES}

Los autores declaran no presentar conflictos de intereses en relación con la preparación y publicación de este artículo.

\section{BIBLIOGRAFÍA}

1. OMS. Salud 21. El marco político de salud para todos de la Región Europea de la OMS. En: Federación Andaluza de Municipios y Provincias [en línea] [consultado el 08/09/2015]. Disponible en www.famp. es/racs/intranet/otras_secciones/documentos/ SALUD\%2021.pdf

2. Avery L, Lazdane G. What do we know about the sexual and reproductive health of adolescets in Europe? Eur J Contracept Reprod Health Care. 2008; 13:58-70

3. Godeau E, Nic Gabhainn S, Vignes C, Ross J, Boyce W, Toodd J. Contraceptive use by 15 -year-old students at their last sexual intercourse: results from 24 countries. Arch Pediatr Adolesc Med. 2008;162:66-73.

4. Ellison MA. Authoritative knowledge and single women's unintentional pregnancies, abortions, adoptions and single motherhood. Social stigma and structural violence. Med Anthropol O. 2003;17:322-47.

5. Estrategia mundial de prevención y control de las infecciones de transmisión sexual 2006-2015: romper la cadena de transmisión. En: OMS [en línea] [consultado el 08/09/2015]. Disponible en apps.who.int/ iris/bitstream/10665/43773/1/9789243563473 spa.pdf

6. Public choices, private decisions: sexual and reproductive health and the millennium development goals. En: UN Millennium Project [en línea] [consultado el 08/09/2015]. Disponible en www.unmillen niumproject.org/documents/MP_Sexual_Health_ screen-final.pdf

7. Brooks F, Van der Sluijs W, Klemera E, Morgan A, Magnusson J, Gabhainn SC, et al. Young people's health in Great Britain and Ireland. Findings from the Health Behaviour in School-Aged Children Study. HBSC International Coordinating Centre. Edimburgo: University of Edinburgh; 2006.

8. Adolescent questionnaire. California Health Interview Survey 2010. En: Universidad de California

\section{ABREVIATURAS}

ESO: Enseñanza Secundaria Obligatoria • ETS: enfermedades de transmisión sexual • ITS: infecciones de transmisión sexual • OMS: Organización Mundial de la Salud • VIH: virus de la inmunodeficiencia humana.

[en línea] [consultado el 01/08/2015]. Disponible en www.chis.ucla.edu

9. Moreno-Rodríguez C, Muñoz Tinoco V, Pérez Moreno PJ, Sánchez Queija I, Granado Alcón MC, Ramos Valverde $\mathrm{P}$, et al. Desarrollo adolescente y salud. Resultados del estudio HBSC 2006 con chicos y chicas españoles de 11-17 años. Madrid: Ministerio de Sanidad y Consumo; 2008.

10. Nebot M, Pérez A, García-Continente X, Ariza C, Espelt A, Pasarín M, et al. Informe FRESC 2008. Resultats principals. Barcelona: Agència de Salut Pública de Barcelona; 2010.

11. Dirección General de Salud Pública y Participación de la Consejería de Salud y Servicios Sanitarios del Principado de Asturias. Encuesta de Salud Infantil en Asturias, 2009. Observatorio de la Infancia y la Adolescencia del Principado de Asturias [en línea] [consultado el 08/09/2015]. Disponible en www.observatoriodela infanciadeasturias.es/cifras_02

12. Schiaffino A, Moncada A, Martín A. Estudi EMCSAT 2008. Conductes de salut de la població adolescent de Terrassa. En: Sidastudi.org [en línea] [consultado el 08/09/2015]. Disponible en http://goo.gl/f1Ouzn

13. Encuesta de Salud del País Vasco, 2007. En: Osakidetza [en línea] [consultado el 08/09/2015]. Disponible en http://goo.gl/GD50HN

14. Servicio de Epidemiología. Hábitos de salud en la población juvenil de la Comunidad de Madrid. Año 2008. Bol Epidemiol Com Madrid. 2009;15:3-48.

15. Encuesta Estatal sobre Consumo de Drogas entre Estudiantes de Enseñanza Secundaria de 14 a 18 años (ESTUDES), 2011. Ministerio de Sanidad y Consumo; 2011.

16. Díez-Gañán L. Hábitos de salud en la población juvenil de la Comunidad de Madrid 2012. Resultados del sistema de vigilancia de factores de riesgo asociados a enfermedades no transmisibles en población juvenil (SIVFRENT-J). Año 2012. Bol Epidemiol Com Madrid. Madrid: Consejería de Sanidad; 2013. Disponible en http://goo.gl/YDOJeg 
17. Informe Juventud en España. Madrid, 2012. En: Instituto de la juventud (INJUVE) [en línea] [consultado el 08/09/2015]. Disponible en http://goo.gl/ R8P7j5

18. Belza MJ, Koerting A, Suárez M, Álvarez R, López M, Melero I, Royo A. Jóvenes, relaciones sexuales y riesgo de infección por VIH/sida en España 2003. FIPSE [en línea] [consultado el 08/09/2015]. Disponible en http://fipse.es/sites/default/files/documentos/pu blicacion/2015/07/20/manual jovenes.pdf

19. Secretaría del Plan Nacional sobre el SIDA. Encuesta de salud y Hábitos Sexuales. España, 2003. En: Instituto Nacional de Estadística [en línea] [consultado el 08/09/2015]. Disponible en http://goo.gl/xwlmfe

20. Estudio sociológico: contexto de la interrupción voluntaria del embarazo en población adolescente y juventud temprana 2006. En: Observatorio de Salud de la Mujer. [en línea] [consultado el 08/09/2015]. Disponible en http://goo.gl/7hH4Q-p

21. Informe 2004. Volumen II: prácticas y comportamientos relativos a los hábitos saludables 2004. Madrid: Instituto de Salud Pública de Madrid [en línea] [consultado el 08/09/2015]. Disponible en http://goo.gl/veLish

22. Salud sexual y reproductiva. Ministerio de Sanidad y Política Social [en línea] [consultado el 08/09/2015] Disponible en www.msssi.gob.es/campannas/cam panas06/enlacesSaludsexual.htm
23. De Irala J, Osorio A, López-del Burgo C, Belen VA, de Guzman FO, Calatrava M, et al. Relationships, love and sexuality: what the Filipino teens think and feel. BMC Public Health. 2009;9:282.

24. Salganicoff A, Wentworth B, Ranji U. Emergency contracepcion in California. Kaiser Family Foundation Survey; 2003. En: Kaiser Family Foundation Social [en línea] [consultado el 08/09/2015]. Disponible en https://goo.gl/k9jsyv

25. Lara Hortega F, Heras Sevilla D. Conocimientos y creencias en la primera etapa de la adolescencia. Datos obtenidos en una muestra de 2.ำ y 3. de ESO de Burgos. INFAD Rev Psicología. 2008;1:249-58.

26. González Hernando C, Sánchez-Crespo Bolaños JR, González Hernando A. Educación integral en sexualidad y anticoncepción para los/las jovenes. Enferm Clin. 2009;19:221-4.

27. Rivadeneyra R, Lebo MJ. Association between television-viewing behaviors and adolescent dating role attitudes and behaviors. J Adolesc. 2008;31:291-305.

28. Shafii T, Burstein GR. An overview of sexually transmitted infections among adolescents. Adolesc Med Clin. 2004;15:201-14. 